thioridazine has been restricted to second-line use in psychosis (Breckenridge, 2000) and the manufacturers, because of these dangers, have withdrawn droperidol.

In May 2000 we carried out a survey of prescribing among psychiatrists in Newcastle. A questionnaire with a typical presentation of an acutely agitated young male was circulated to all psychiatrists. Respondents were asked for preferred first- and second-line management regimes. The response rate was $48 \%$. Seventy-eight per cent of respondents indicated that droperidol plus lorazepam would be their first choice, followed by zuclopenthixol acetate (9\%) and others $(15 \%)$. The second-line choices were zuclopenthixol acetate (28\%), droperidol (28\%), lorazepam (28\%) and others (16\%).

If these results represent common practice in adult psychiatry, there is cause for concern. Withdrawal of droperidol therefore requires urgent revision of guidelines regarding acute agitation and consideration of alternatives. These could include olanzapine in intramuscular form (currently being used experimentally) and the more widespread use of intravenous sedation protocols. However, using the above would have considerable implications for already hard-pressed drug budgets and the level and skill of nursing observation available.

BRECKENRIDGE, A. (2000) Thioridazine: restricted indications and new warnings on cardiotoxicity. Committee on Safety of Medicines.

http://www.open.gov.uk/mca/ourwork/ monitorsafequalmed/safetymessages/thiolet.

REILLY, J. G., AVIS, S. A., FERRIER, N., et al (2000) QTCinterval abnormalities and psychotropic drug therapy in psychiatric patients. Lancet, 355, 1048-1052.

J. P. Richardson Clinical Research Associate S. Joseph Consultant Psychiatrist, Wolfson Research Centre, Newcastle General Hospital, Westgate Road, Newcastle-upon-Tyne NE4 8BE

\section{Hyoscine patches in clozapine-induced hypersalivation}

Sir: Hypersalivation associated with clozapine can be a distressing and difficult to manage side-effect (Cree et al, Psychiatric Bulletin, 2001, 25, 114-116). In recent months we have used hyoscine hydrobromide patches in a group of patients with disabling hypersalivation. We had used the patches to treat severe hypersalivation in a patient on depot medication on the advice of a colleague working in ear, nose and throat medicine. This patient had gained some relief from atropine drops but could not tolerate the bitter taste. He had not been helped by oral anticholinergic medication and it was to our surprise that he responded dramatically to the use of the patches.
We have since used the patches on four patients with severe disabling, clozapineinduced hypersalivation (dosage 500-800 mg/day). These patients had obvious drooling that often required a change of clothing with marked soaking of their pillows each morning. A dramatic improvement has been noted in each case.

The patches are well tolerated. They are sited on bare skin behind the ear and release $1 \mathrm{mg}$ of hyoscine over a 72-hour period. They have brought about a sustained improvement in our patients' quality of life. This small sample suggests that hyoscine patches are effective especially in those patients suffering severe hypersalivation. It is interesting to note that the patches proved effective in patients who had not responded to oral treatments, including oral hyoscine.

J.P. McKane Consultant Psychiatrist,

Charlotte Hall Senior House Officer, Gazala Akram Clinical Pharmacist, Leverndale Hospital, 510 Crookston Road, Glasgow

\section{Patient or client?}

Sir: The article by Ritchie et al (Psychiatric Bulletin, December 2000, 24, 447-450) provides an 'evidence base' for a debate that has been going on for some time now. It confirms the beliefs of many clinicians, that patients prefer to be called patients. We have so far refrained from using the words 'client' or 'service user' without any problems.

The origins of the word patient go back to the 14th century and are derived from old french, from the latin word 'patiens' meaning one who is suffering. Webster's Dictionary defines patient as an "individual awaiting, or under medical treatment". Client is defined as a "customer or patron, or one who depends on the protection of another". By these definitions, it is the word patient that best suits the people under our care.

As far as we are aware, it is only patients under psychiatric care who face these dilemmas in nomenclature. People receiving general medical care are still called patients. By calling patients under psychiatric care anything different and by denying that they too suffer from a medical illness, we only minimise their suffering and stigmatise them further.

Let us not deny that they are going through pain and suffering. There is enough stigma attached to suffering from a mental illness. Let us not segregate them further. Our patients have expressed what they wish to be called. For once, let us listen to them.

Maju Mathews Specialist Registrar in Psychiatry Mental Health Unit, Basildon Hospital, Basildon SS16 5NL, Joanne Mathews Senior House Officer in Psychiatry, Warley Hospital, Brentwood (e-mail majumathews@yahoo.co.uk)
Sir: We read with interest the article by Ritchie et al (Psychiatric Bulletin, December 2000, 24, 447-450) describing the preferences for the terms patient and client with respect to people attending a psychiatric clinic. Our prior study (Sharma et al, 2000), which involved a survey of 550 service providers and 427 service recipients at four sites in Canada - two provincial psychiatric hospitals, a private mental health centre and a psychiatric unit of a general hospital - showed similar results. The term patient was favoured by both service providers (68.4\%) and service recipients (54.8\%). The logistic regression results for service recipients' preference for the term patient showed that the variables of site, diagnosis and employment status contributed the most to prediction. More specifically, service recipients from the rural provincial psychiatric hospital had lower odds of preferring the term patient than did service recipients from the urban provincial psychiatric hospital. Service recipients with the diagnosis of mood disorders had higher odds of preferring the term patient than those with substance misuse disorders. Retired service recipients were more likely to prefer the term patient than those who were employed (Sharma et al, 2000).

The consensus among providers and recipients in current publications favours the use of the word patient. In our view the word patient carries a broader and perhaps deeper fiduciary commitment than does client.

SHARMA, V.,WHITNEY, D., KAZARIAN, S., et al (2000) Preferred terms for users of mental health services among service providers and recipients. Psychiatric Services, 51, 203-209.

*Verinder Sharma Mood Disorders Program, Regional Mental Health Care London, 850 Highbury Avenue North, London, Ontario, Canada N6A $4 \mathrm{H1}$ ， Shahé S. Kazarian Diane Whitney Rahul Manchanda St Joseph's Health Care, London

\section{Leisure activities of people with schizophrenia: listening to music and playing the National Lottery}

Sir: Although much has been written about the behavioural aspects of people with schizophrenia living in the community (Leff \& Trieman, 2000), little is known about their leisure activities. We recently asked patients and normal subjects in Nithsdale, South West Scotland, about their access to music ( $n=136$ patients and 114 controls) and the National Lottery ( $n=62$ patients and 57 controls).

More controls owned a cassette $(96 \%$ v. $73 \%)$, record $(60 \%$ v. $40 \%)$ or CD $(85 \%$ v. $52 \%$ ) player; patients owned fewer cassettes, records or CDs. More patients 